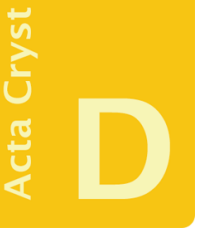

BIOLOGICAL

\title{
CRYSTALLOGRAPHY
}

Volume 71 (2015)

Supporting information for article:

Structural and functional analysis of Hikeshi, a new nuclear transport receptor of Hsp70

Jinsue Song, Shingo Kose, Ai Watanabe, Se-Young Son, Saehae Choi, Hyerim Hong, Eiki Yamashita, II Yeong Park, Naoko Imamoto and Soo Jae Lee 


\section{S1. Methods}

\section{S1.1. Plasmid construct}

Human Hsc70 fragments except for full-length Hsc70 were cloned into bacterial expression vector pET28a (Novagen) modified to a TEV cleavage site with NdeI/XhoI. Human full-length Hsc70 was cloned into the pQE80L expression vector (Qiagen) with BamHI/SalI. Human Hikeshi and its mutants were cloned into the entry vector, pENTR (Invitrogen). Point and deletion mutants were produced by PCR-based mutagenesis. To construct the Gateway destination vector, the PCR fragment containing PreScission protease site-FLAG-RfB (RfB; Invitrogen) was inserted into the NdeI-XhoI site of pColdProS2 vector (Takara). The resultant vector is referred to as the pCold-ProS2-PP-FLAG-GW vector. Expression plasmids for the 6xHis-ProS2-FLAG-tag-fused Hikeshi proteins were constructed using Gateway technology. All plasmid inserts were verified by DNA sequencing.

\section{S1.2. Expression and purification of recombinant proteins}

The recombinant Hsc70 fragments for the pull-down assay were expressed in BL21(DE3) cells (Stratagene) by $0.3 \mathrm{mM}$ IPTG induction at $18{ }^{\circ} \mathrm{C}$ and purified from soluble fraction by Ni-NTA agarose (Qiagen). The purified proteins were quickly exchanged for transport buffer. The expression and purification of recombinant GST- and GFP-fusion Hikeshi proteins and GFP-Hsc70 proteins were performed as described previously (Kose et al., 1997, Kose et al., 2012). To express 6xHis-ProS2FLAG-tag-fused proteins, the respective expression plasmids were transformed in E. coli BL21. The resulting E. coli cells were grown at $37^{\circ} \mathrm{C}$ in $\mathrm{LB}$ medium containing $50 \mathrm{mg} / \mathrm{mL}$ ampicillin. After leaving to stand in culture medium for $30 \mathrm{~min}$ at $4{ }^{\circ} \mathrm{C}$, protein expression was induced with $0.1 \mathrm{mM}$ IPTG, followed by shaking culture for $18 \mathrm{~h}$ at $15^{\circ} \mathrm{C}$. Cultures were harvested by centrifugation and resuspended in lysis buffer (50 mM Tris- $\mathrm{HCl}, \mathrm{pH} 8.3,500 \mathrm{mM} \mathrm{NaCl}, 20 \mathrm{mM}$ 2-mercaptoethanol, 0.2 mM PMSF). After cell disruption by freeze-thaw and sonication, the extract was clarified by centrifugation and incubated with Ni-NTA agarose (QIAGEN) at $4{ }^{\circ} \mathrm{C}$. After washing with lysis buffer containing $20 \mathrm{mM}$ imidazole, recombinant proteins were eluted with $50 \mathrm{mM}$ Tris-HCl, $\mathrm{pH} 7.3$, $50 \mathrm{mM} \mathrm{NaCl}, 20 \mathrm{mM}$ 2-mercaptoethanol, $250 \mathrm{mM}$ imidazole or digested with PreScission protease for cleavage of 6xHis-ProS2-tag at $4{ }^{\circ} \mathrm{C}$. The recombinant proteins were further purified on Hi-TrapQ column (GE Healthcare) using a linear gradient elution of $50-500 \mathrm{mM} \mathrm{NaCl}$ in $50 \mathrm{mM}$ Tris- $\mathrm{HCl}$, pH 7.3, 1 mM DTT, and peak fractions were dialyzed against transport buffer (TB; $20 \mathrm{mM} \mathrm{HEPES,} \mathrm{pH}$ 7.3, $110 \mathrm{mM}$ potassium acetate, $2 \mathrm{mM}$ magnesium acetate, $5 \mathrm{mM}$ sodium acetate, $1 \mathrm{mM}$ EGTA)/1 mM DTT, and concentrated using AmiconUltra-4 (Amicon). 


\section{References}

Kose S, Furuta M, \& Imamoto N (2012). Cell 149. 578-589.

Kose S, Imamoto N, Tachibana T, Shimamoto T, \& Yoneda Y (1997). The Journal of cell biology 139, 841-849. 
Table S1 Hydrophobic interactions within 5 angstroms observed in dimer interface via CTD between monomers A and B.

\begin{tabular}{|c|c|c|c|c|c|}
\hline Residue & Position & Chain & Residue & Position & Chain \\
\hline Phe & 138 & $\mathrm{~A}$ & Val & 132 & B \\
\hline Phe & 138 & A & Phe & 138 & B \\
\hline Phe & 138 & A & Phe & 141 & B \\
\hline Phe & 141 & A & Phe & 138 & B \\
\hline Phe & 141 & A & Phe & 141 & B \\
\hline Phe & 141 & A & Phe & 184 & B \\
\hline Phe & 141 & A & Trp & 195 & B \\
\hline Phe & 149 & A & Leu & 146 & B \\
\hline Phe & 149 & A & Phe & 149 & B \\
\hline Phe & 149 & A & Val & 176 & B \\
\hline Phe & 149 & A & Val & 177 & B \\
\hline Phe & 149 & A & Trp & 195 & B \\
\hline Phe & 152 & A & Val & 176 & B \\
\hline Phe & 152 & A & Trp & 180 & B \\
\hline Ala & 153 & A & Ile & 172 & B \\
\hline Ala & 153 & A & Val & 176 & B \\
\hline Phe & 156 & A & Pro & 173 & B \\
\hline Phe & 156 & A & Val & 176 & B \\
\hline Ala & 157 & A & Ile & 172 & B \\
\hline Ala & 161 & A & Pro & 167 & B \\
\hline Phe & 171 & A & Val & 158 & B \\
\hline Phe & 171 & A & Phe & 171 & B \\
\hline Phe & 171 & A & Pro & 173 & B \\
\hline Ile & 172 & A & Phe & 149 & B \\
\hline Ile & 172 & A & Ala & 153 & B \\
\hline Ile & 172 & A & Ala & 157 & B \\
\hline Ile & 172 & A & Ile & 172 & B \\
\hline
\end{tabular}




\begin{tabular}{|c|c|c|c|c|c|}
\hline Ile & 172 & A & Ala & 174 & B \\
\hline Pro & 173 & A & Phe & 156 & B \\
\hline Pro & 173 & A & Phe & 171 & B \\
\hline Ala & 174 & A & Ile & 172 & B \\
\hline Val & 176 & A & Phe & 152 & B \\
\hline Val & 176 & A & Ala & 153 & B \\
\hline Val & 176 & A & Phe & 156 & B \\
\hline Val & 177 & A & Phe & 149 & B \\
\hline Val & 177 & A & Ile & 172 & B \\
\hline $\operatorname{Trp}$ & 180 & A & Phe & 149 & B \\
\hline $\operatorname{Trp}$ & 180 & A & Phe & 152 & B \\
\hline Phe & 184 & A & Phe & 141 & B \\
\hline Leu & 188 & A & Phe & 141 & B \\
\hline $\operatorname{Trp}$ & 195 & A & Phe & 141 & B \\
\hline
\end{tabular}



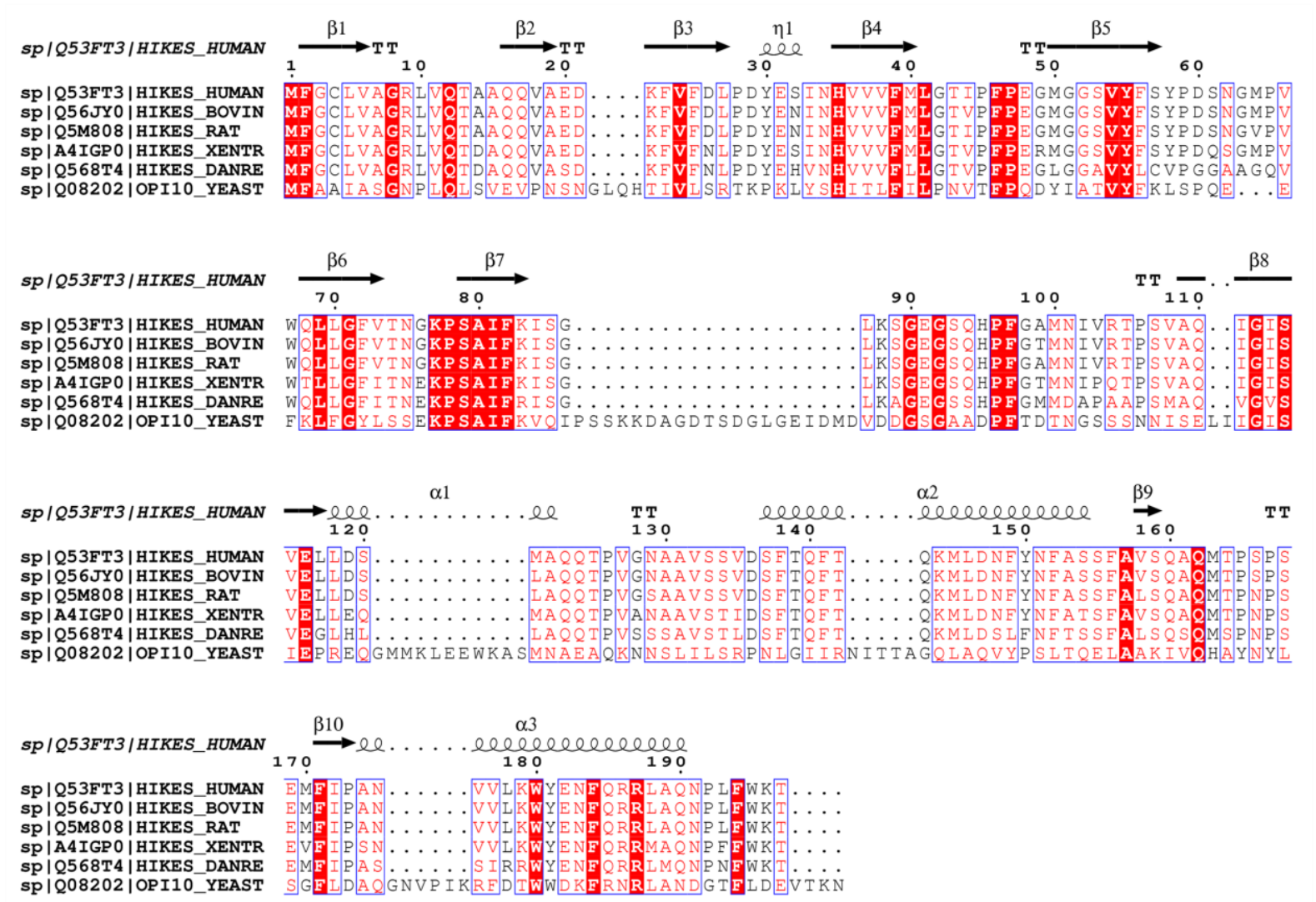

Figure S1 Alignment of Hikeshi protein sequences. Amino acid sequences of selected Hikeshi homologs were aligned using the EBI Clustal-X server. Secondary structure elements for Hikeshi from human are indicated above the sequences by black coloring. The Uniprot codes for the sequences are: Q53FT3, Homo sapiens (Human); Q56JY0, Bos taurus (Cattle); Q5M808, Rattus norvegicus (Rat); A4IGP0, Xenopus tropicalis (African clawed frog); Q568T4, Danio rerio (Zebrafish); Q08202, Saccharomyces cerevisiae (Yeast). 

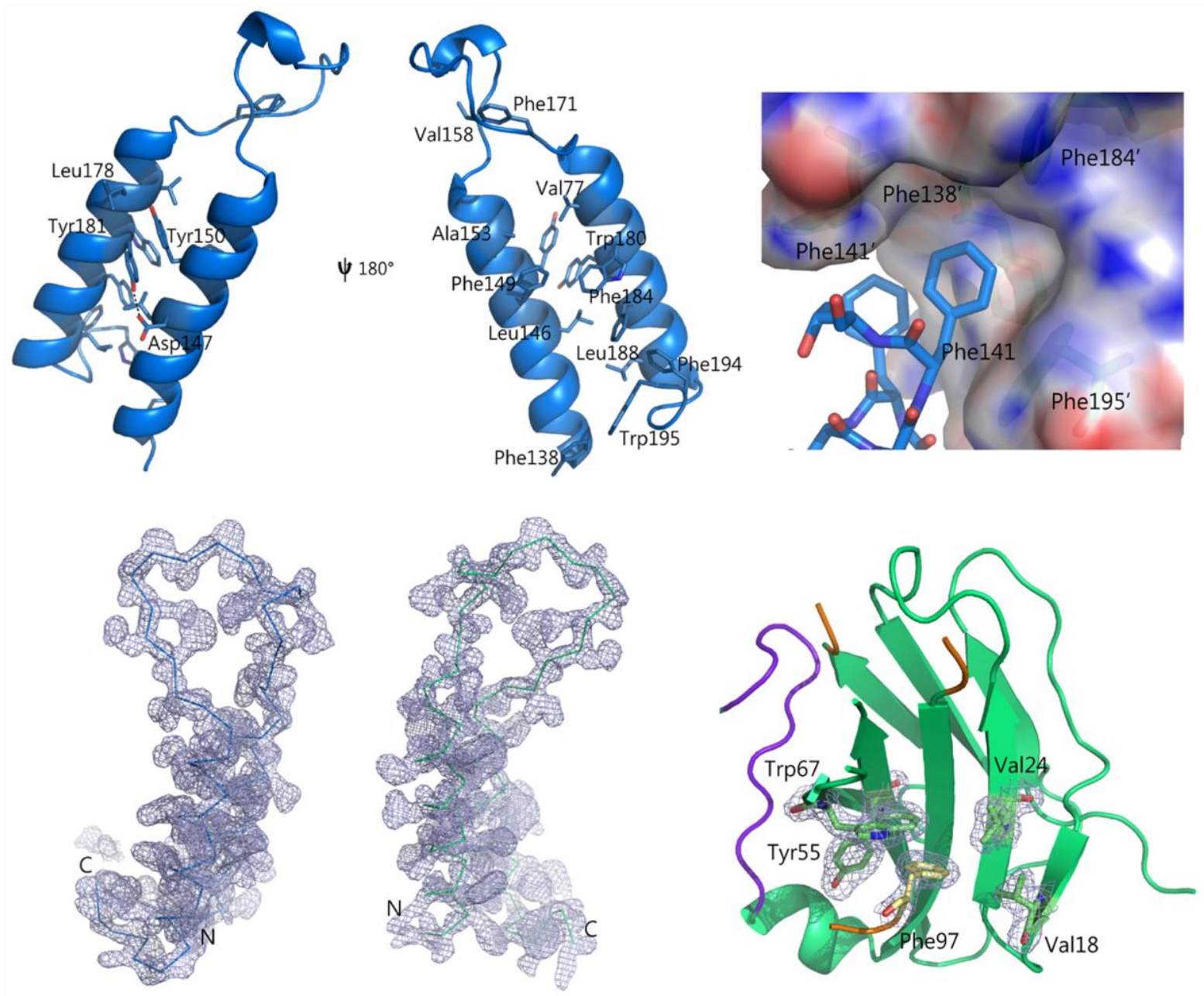

Figure S2 Structural analysis and quality of domain crossover of Hikeshi. (A) Structure of the CTD in monomer A. Front view of the helical hairpin monomer showing residues in the intramolecular interface of two antiparallel helices $(\alpha 2, \alpha 4)$ and a flexible segment connecting $\alpha 2$ and $\alpha 4$. The amino acids forming the hydrophobic core are indicated as sticks. Hydrogen bond interactions are denoted by black dotted lines (left, colored as in Fig. 1a). A flipped view of the same structure in a, roughly related by a $180^{\circ}$ rotation (right). (B) Phe141, shown as sticks (O, red; $\mathrm{N}$, blue) lodging in the hydrophobic core formed by residues Phe138, Phe141, Phe184, and W195 on another monomer (surface representation). (C) Stereo view of the domain crossover region (left: monomer A; right: monomer B) of CTDs in the reconstructed density map (violet mesh; $1 \sigma$ threshold) with an atomic model (ribbon diagram). (D) View of the E-loop and hydrophobic pocket in the NTD of monomer B shown as cartoon representation. It is evident that Phe97A is buried in the hydrophobic pocket although the rest of the E-loop is disordered. The color code is the same as in Fig. 1a. 


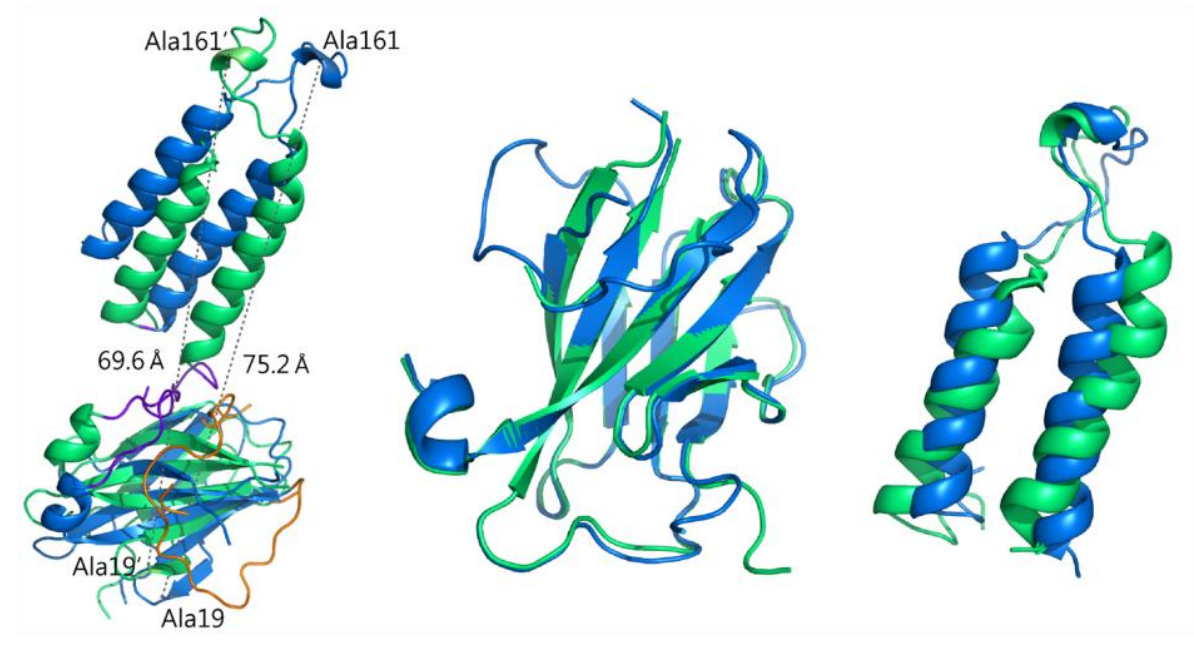

Figure S3 Structural overlay of both monomers from the Hikeshi dimer. (A) Superposition of the full-length structure. The calculated r.m.s.d. of both monomers is $6.7 \AA$ for $193 \mathrm{C} \alpha$ atoms. In addition, the distances between Ala19- and Ala161-C $\alpha$ atoms of each monomer, being on each end of the longest axis of the molecule, are $69.6 \AA$ and $75.2 \AA$, respectively. (B) Superposition of the NTDs. The NTDs have a high overall topological similarity with an r.m.s.d. of $0.5 \AA$ in $123 \mathrm{C} \alpha$ atoms. (C) Superposition of CTDs showed a remarkable difference with a large r.m.s.d. of $3.1 \AA$ in $60 \mathrm{C} \alpha$ atoms. 

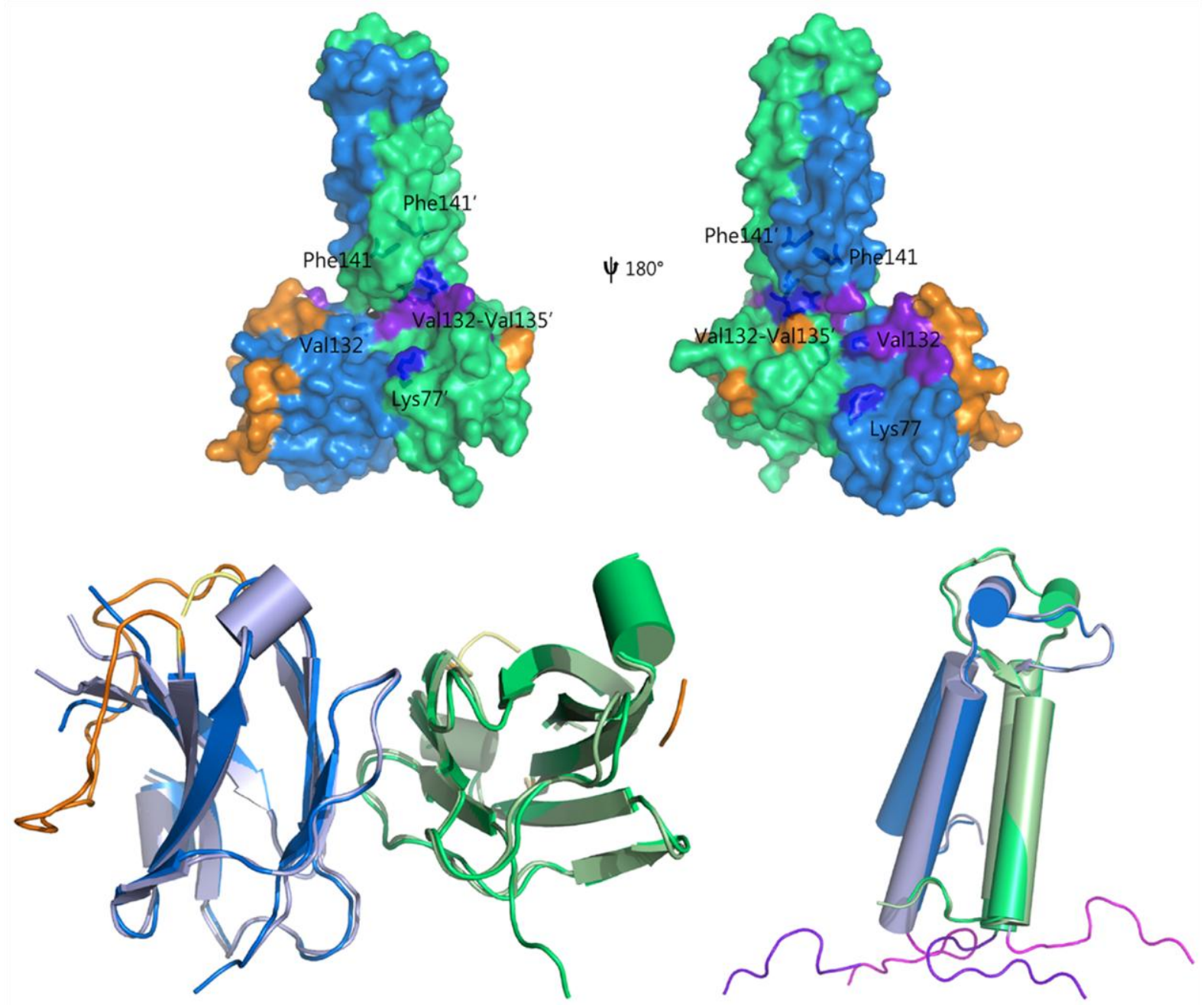

Figure S4 (A) Surface representation showing Hsp70 binding-related residues and region (in blue color). The color code of the secondary structure elements is the same as in Fig. 1A. (B) and (C) Superposition of NTDs of wt and Phe97Ala. The NTDs and CTDs have a high overall topological similarity with an r.m.s.d. of $0.57 \AA$ and $0.45 \AA$, respectively. 

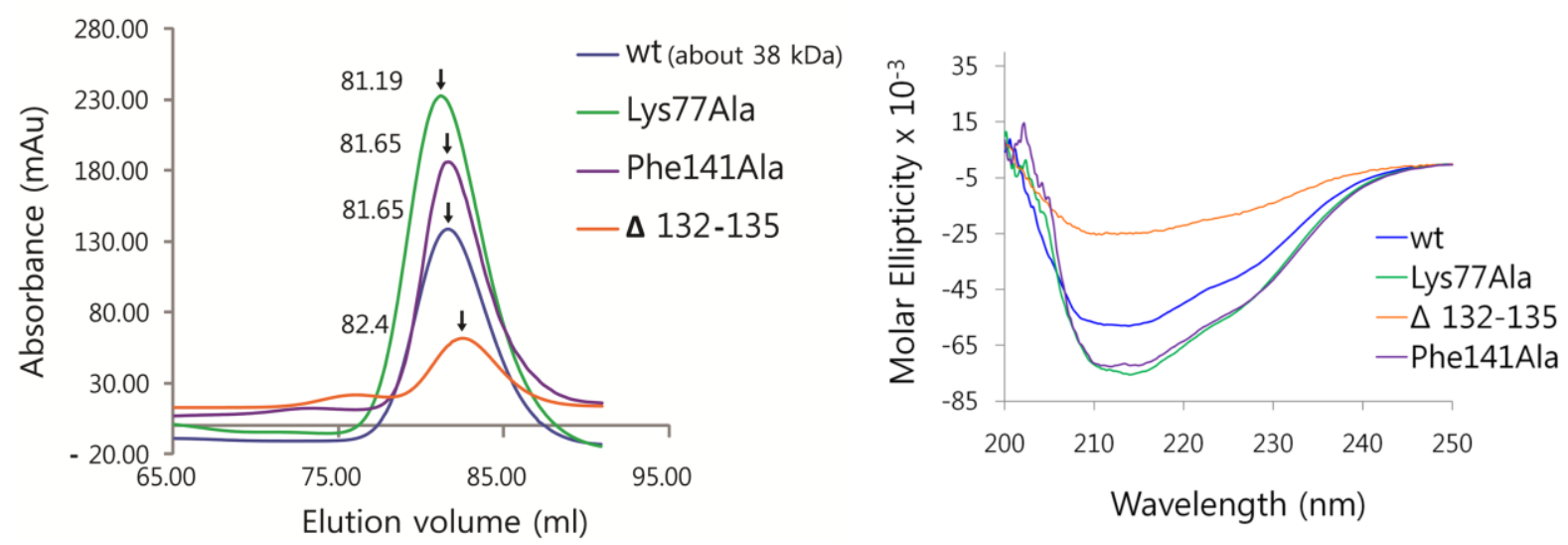

Figure S5 (A) The profiles of gel filtration for the mutant Hikeshi proteins. Gel filtration was performed with a HiLoad 16/60 Superdex 200 (GE Pharmacia) in $20 \mathrm{mM}$ Tris-HCl (pH 8.0), $20 \mathrm{mM}$ $\mathrm{NaCl}$, and $1 \mathrm{mM}$ DTT. Black arrows indicate the top of eluted peak. (B) The secondary structures of the Hikeshi mutants were detected by $\mathrm{CD}$. The characteristic shape and magnitude of the CD spectral trace are all similar to those of wt Hikeshi. Five scans were averaged for each mutant or the wt protein. 

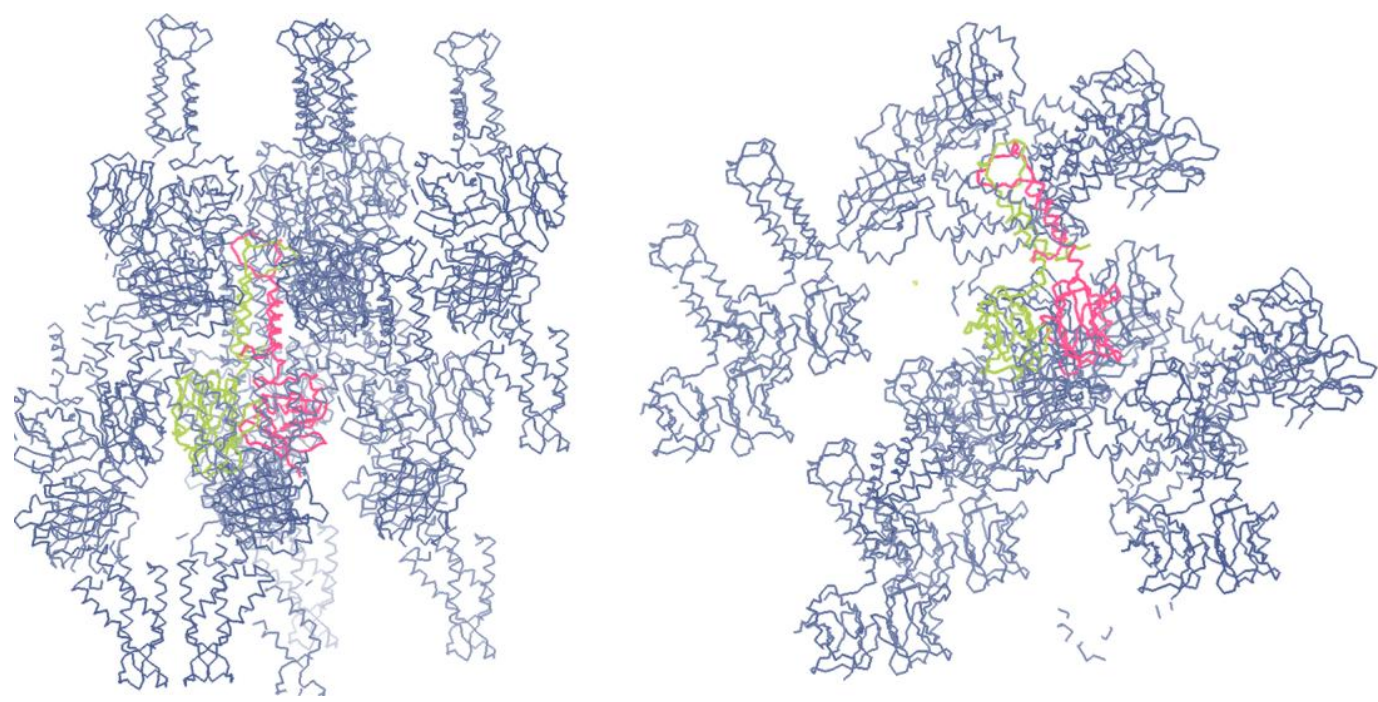

Figure S6 Crystal lattices of wt and Phe97Ala mutant structures in the $C 222_{1}$ (left) and $P 3_{2}$ (right) space groups, respectively. 


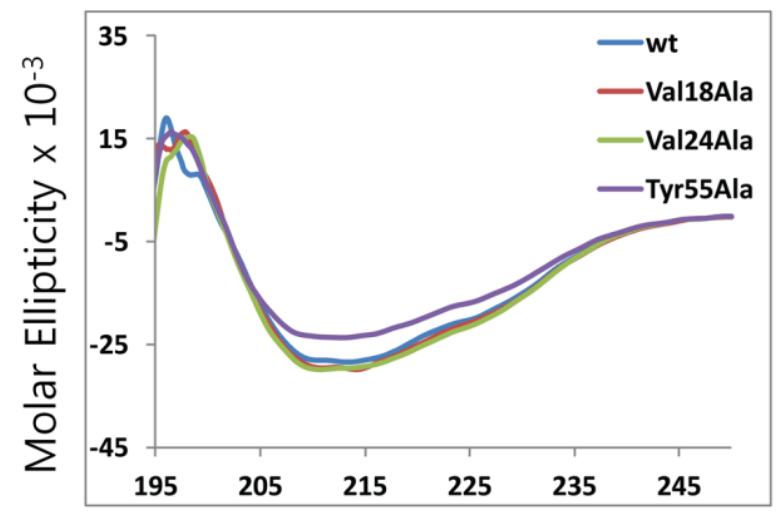

Wavelength $(\mathrm{nm})$

Figure S7 The secondary structures of the Hikeshi mutants were detected by CD. The characteristic shape and magnitude of the CD spectral trace are all similar to those of wt Hikeshi 